\title{
COMPORTAMIENTO AL SECADO NATURAL DE CUATRO ESPE CIES MADERABLES DE BOSQUES SECUNDARIOS
}

\section{Leticia Guevara Salnicov*}

\section{RESUMEN}

Con la finalidad de estudiar el comportamiento al secado natural de cuatro especies maderables de bosques secundarios, se efectuaron los ensayos pertinentes en las siguientes maderas:

Trema micrantha (atadijo), Sapium marmieri Hubert (caucho masha), J acaranda copaia Aube. D. Don (huamazamana) y Ochroma pyramidale Swartz (topa). Los ensayos se efectuaron de acuerdo a las especificaciones técnicas propuestas por A róstegui et. al. (1975). Se ensayaron tablas de $2 \times 15 \times 120 \mathrm{~cm}$, simplemente aserradas, rotuladas y con los extremos pintados con barniz sintético. Se apilaron horizontalmente sobre bases de madera a $35 \mathrm{~cm}$ del nivel del suelo y usando separadores de madera seca al aire y con un espesor de $2 \mathrm{~cm}$. El contenido de humedad inicial se determinó en probetas de control por el método de secado en estufa hasta peso constante. En las tablas de ensayo se determinó la variación del contenido de humedad cada quince días mediante la variación del peso. Una vez que se tuvo un contenido de humedad por debajo del $20 \%$, se procedió a evaluar defectos (grietas y rajaduras) y deformaciones (arqueadura, encorvadura, torcedura y revirado). L os resultados se interpretaron de acuerdo a las especificaciones propuestas por A róstegui (1979). De acuerdo a los resultados se concluye que las cuatro maderas ensayadas en las dimensiones especificadas secan rápidamente al aire, la formación de defectos y deformaciones no es pronunciada.

\footnotetext{
* Ing. Forestal. Investigadora IIAP en tecnología de Maderas
} 


\section{INTRODUCCION}

A ctual mente la industria maderera nacional atraviesa una seria crisis debida, entre otras causas, al alto costo de extracción de la materia prima en los bosques primarios y al escaso valor agregado de los productos.

Una estrategia para superar esta crisis es el aprovechamiento de los bosques secundarios, en los que existen numerosas especies maderables de rápido crecimiento, actualmente no aprovechadas, debido al desconocimiento de sus propiedades tecnológicas. Para usos comunes, especialmente para interiores y manufacturas de acabado superficial, el secado es una operación indispensable, que permite la estabilidad dimensional de la madera y la compatibilidad con productos y procesos de tratamiento preservador y de acabado.

El objetivo del presente trabajo de investigación es estudiar el comportamiento de cuatro maderas de bosques secundarios al secado natural. El estudio forma parte del plan de trabajo del Proyecto Estudios Básicos y Tecnológicos de M aderas de Bosques Secundarios que ejecuta el IIAP en convenio con el INIA y la UNU. Los ensayos se efectuaron de acuerdo a las especificaciones técnicas propuestas por A róstegui et al (1975), en la Estación Experimental Pucallpa del INIA. Se estudiaron las siguientes especies: Trema micrantha (atadijo), Sapium marmieri Hubert, (caucho masha), Jacaranda copaia Aube D. Don (huamazamana) y O chroma pyramidale Swartz (topa). Los resultados indican que de acuerdo a las condiciones del ensayo, básicamente a las dimensiones de las tablas y condiciones atmosféricas predominantes entre los meses de julio a octubre, las maderas estudiadas secan rápidamente y sin formación de defectos y deformaciones en forma pronunciada.

\section{REVISION DE LITERATURA}

La madera recién aserrada contiene cantidades variables de agua, que depende de la época de corta, la región de procedencia, la especie forestal, la edad del árbol, entre otros factores, según JUNAC (1984). En general, las maderas livianas contienen mayor cantidad de agua que las maderas pesadas. La albura, formada por células cuya principal función es la conducción de sustancias nutritivas en solución acuosa, presenta un contenido de humedad mayor que el duramen, tejido compuesto por células con acumulación de sustancias infiltradas en las cavidades celulares. 
Según Gonzáles (1970), el agua susceptible de ser eliminada por secado presenta dos formas. El agua libre ocupa las cavidades celulares o lumen de los el ementos vasculares y depende linealmente de la densidad básica, la que a su vez es función directa del volumen de poros, según la siguiente ecuación:

$$
\text { CHmax }=\frac{1,56-D B}{(1,56)(D B)} \quad 100 \%
$$

Donde:

CH max es el máximo contenido de humedad, expresado en porcentaje; DB es la densidad básica o relación del peso seco en estufa y el volumen saturado, determinado en un ensayo normalizado de laboratorio, expresado en $\mathrm{g} / \mathrm{cm}^{3} ; 1,56$ es un valor constante asignado como la densidad real de los principales componentes de la madera, básicamente celulosa.

Este tipo de agua se pierde fácilmente por evaporación. Según Hildebrant (1967) la velocidad de eliminación del agua libre depende de las condiciones del medio, básicamente de la humedad relativa y la temperatura. También depende del diámetro de las cavidades celulares, que define las fuerzas de atracción capilar. En los puntos en que se ha evaporado el agua libre se inicia la difusión del agua higroscópica en forma de vapor de la pared celular a la cavidad celular, en donde se condensa en forma líquida.

El agua higroscópica tiene especial relevancia, ya que durante su eliminación se inicia la contracción de la madera. Según Siau, citado por JUNAC (1984), el margen del punto de saturación de la fibra está entre 21 a 32\% para la mayor parte de las maderas de latifoliadas. Por debajo del punto de saturación de la fibra se produce la migración de la humedad en virtud de las diferencias de presión parcial de vapor de agua, con predominio de la difusión de vapor de agua por las paredes celulares y los poros, asi como por las cavidades celulares. A temperatura constante la cantidad de agua difundida depende de la gradiente de humedad; con elevación de la temperatura aumenta la difusión del vapor de agua $y$, consecuentemente, aumenta la velocidad de secado. 
Los factores que influyen en el comportamiento de la madera durante el secado dependen fundamentalmente de la anatomía de la madera. Según Gonzales (1970) la dirección del grano o disposición de los elementos estructurales de la madera influye en la velocidad de secado y en la formación de defectos y deformaciones. De acuerdo con Hunt y Garrat (1966), a través de las perforaciones y de los poros se verifica la circulación longitudinal y axial de líquidos y gases. Su eficacia depende de estar libre de inclusiones y de tilosis y de la permeabilidad de los tejidos circundantes, así como el tamaño, número y distribución. En las maderas de poros difusos, los poros son aproximadamente iguales y están regularmente dispersos, por tanto la circulación de agua tiende a ser más rápida. Las fibras no son muy aptas para la conducción de líquidos, aunque su permeabilidad relativa puede tener notable influencia en la extensión subsiguiente de líquidos procedentes de los vasos. El parénquima, tanto el leñoso como el radial, no favorece la circulación de los líquidos, debido a la presencia de poros simples, pero facilita la salida de vapor de agua a través de aberturas finísimas.

De acuerdo con los estudios efectuados por A róstegui et al (1975), la madera de Sapium marmieri Hubert (caucho masha) es de color amarillo, grano recto, textura de media a gruesa. En la estructura anatómica destacan los poros solitarios y múltiples radiales, medianos, sin tilides y con ligeras inclusiones. LoS vasos son de longitud media. Presenta parénquima paratraqueal aliforme confluente. L L as fibras son de longitud media y de diámetro grande. L a densidad es baja, la resistencia a la mayor parte de solicitaciones mecánicas es baja, excepto en la dureza de extremos y lados donde la resistencia es muy baja.

L a madera de J acaranda copaia A ube D. Don (huamanzamana) ha sido estudiada por Valderrama et al (1986). Según este autor, la madera es de color amarillo, grano recto, textura media. Presenta poros difusos, de diámetro de mediano a grande, pocos por mm2. Los vasos son de muy cortos a medianos. El parénquima es predominantemente paratraqueal, aliforma simple y confluente.

Según Detienne (1983) la madera de Ochroma pyramidale Swartz (topa) tiene color rosado, grano recto y textura gruesa. Presenta poros difusos, solitarios y múltiples radiales. El diámetro de los elementos vasculares es clasificado como grande. El parénquima es paratraqueal vasicéntrico. Las fibras son de longitud media a grande, diámetro muy grande y espesor de pared delgada. La densidad es muy baja. 


\section{MATERIALES Y METODOS}

\subsection{L ugar de ejecución}

Los ensayos se efectuaron en el laboratorio de tecnología de la madera de la Estación Experimental Pucallpa - INIA.

\subsection{Maderas estudiadas}

El Cuadro 1 presenta la relación de maderas estudiadas. Las trozas proceden de un bosque secundario situado a la altura del km 18 de la carretera F ederico Basadre, entrando $10 \mathrm{~km}$ por la carretera secundaria.

El bosque tiene una extensión de 7706 has y ha sido adjudicado al Instituto Superior Tecnológico Suiza para fines de investigación.

\section{Cuadro № 1 Relación de especies estudiadas}

\section{Nombre común Nombre científico}

A tadijo

Trema micrantha

Caucho masha

Sapium marmeri Hubert

H uamanzamana

Topa
Jacaranda copaia A ube D. Don

Ochroma pyramidale Swartz
Familia

ANNONACEAE

BOMBACACEAE

BIGNONIACEAE

MORACEAE 


\subsection{Identificación dendrológica}

La identificación taxonómica se efectuó en el laboratorio de dendrología de la Facultad de Ciencias Forestales de la Universidad Nacional de U cayali.

\subsection{Repeticiones}

Se tomaron tres árboles por especie y tres niveles por árbol. En el bosque se ha identificado y marcado árboles coetáneos de las cuatro especies para estudios complementarios y para futuros estudios sobre la influencia de la edad del árbol en las características, propiedades y aptitud tecnológica de la madera.

\subsection{Equipos}

- Balanza eléctrica $0.1 \mathrm{~g}$ de aproximación

- Balanza mecánica $1 \mathrm{~g}$ de aproximación

- Estufa el éctrica con termostato regulable

- M aquinaria de carpintería

- M artillo

\section{PROCEDIMIENTO}

\subsection{Probetas de ensayo}

Las trozas fueron aserradas a $2 \mathrm{~cm}$ de espesor y habilitadas a $15 \mathrm{~cm}$ de ancho y $120 \mathrm{~cm}$ de longitud. Se seleccionaron probetas libres de defectos y signos de biodeterioro. Se rotularon por estarcido y se sellaron los extremos con pintura esmalte sintético. El número de probetas por especie utilizadas para el ensayo se indica en el Cuadro $\mathrm{N} N 2$. 


\section{Cuadro № 2 Probetas ensayadas por árbol de procedencia}

\begin{tabular}{lllll}
\hline M adera & \multicolumn{3}{c}{ Código de ár bol } & T otal \\
& $\mathbf{1}$ & $\mathbf{2}$ & $\mathbf{3}$ & \\
\hline A tadijo & 9 & 9 & 9 & 27 \\
Caucho masha & 7 & 5 & 8 & 20 \\
H uamanzamana & 8 & 7 & 6 & 21 \\
Topa & 9 & 9 & 9 & 27 \\
\hline \multirow{2}{*}{ Total } & 33 & 30 & 32 & 95 \\
\hline
\end{tabular}

\subsection{Contenido de humedad inicial}

Se determinó mediante el método de secado en estufa hasta peso constante de pequeñas probetas aserradas de las tablas utilizadas para preparar las probetas de control. Con esta información se determinó el peso seco calculado, como sigue a continuación:

$$
\begin{aligned}
\mathrm{PSC}= & \frac{\mathrm{PI}}{\mathrm{CH}+100} \quad 100 \% \\
\mathrm{CH}= & \frac{\mathrm{P} 1-\mathrm{P} 2}{\mathrm{P} 2}
\end{aligned}
$$

Donde:

PSC es el peso seco calculado de las probetas de ensayo, expresado en gramos; PI es el peso inicial de las probetas de ensayo, expresado en gramos; CH es el contenido de humedad de las probetas de control, expresado en porcentaje; $\mathbf{P} \mathbf{1}$ es el peso inicial de las probetas de control, expresado en gramos; $\mathbf{P 2}$ es el peso seco a estufa de las probetas de control, expresado en gramos. 


\subsection{A pilado}

Se utilizó el apilado horizontal sobre bases de madera a $35 \mathrm{~cm}$ de altura, sobre las que se colocaron vigas de $10 \mathrm{~cm}$ de altura. Sobre éstas descansaban las probetas de ensayo, entre hileras se colocaron separadores de $2 \times 2.5 \times 120 \mathrm{~cm}$, de madera seca al aire, distanciados $56.25 \mathrm{~cm}$ entre si. Se colocaron seis probetas por hilera y quince por columna. En la parte superior de la pila se colocaron cuartones de madera con la finalidad de ejercer peso y compensar tensiones que provocan deformaciones en las probetas de las últimas hileras.

\subsection{E valuación de la velocidad de secado}

Cada quince días se pesaron las probetas y con los valores del peso seco calculado se determinó el contenido de humedad. El ensayo se dio por terminado cuando todas las probetas estaban por debajo del $20 \%$ de contenido de humedad.

\subsection{Evaluación de defectos y deformaciones}

M ediante inspección visual y con medición directa se evaluaron defectos de secado, básicamente grietas y rajaduras, y deformaciones: arqueadura, encorvadura, revirado y torcedura.

\subsection{Interpretación de resultados}

Según el procedimiento elaborado por A róstegui et. al. y en base a la velocidad de secado, las maderas se clasifican en tres grupos:

Secado rápido, aquellas maderas que alcanzan un contenido de humedad de $20 \%$ en 120 días.

Secado moderado, si llegan al 20\% entre 121-240 días.

Secado lento, cuando llegan al $20 \%$ en más de 240 días.

En función a la presencia y magnitud de defectos y deformaciones, las maderas se clasifican en tres grupos: 
Grupo A: Maderas que secan sin defectos ni deformaciones. Puede admitirse rajaduras en ambos extremos, siempre que no pasen el $1 \%$ de la longitud de la pieza;

Grupo B: Maderas que secan con ligeros defectos y deformaciones. Puede admitirse rajaduras de hasta $5 \%$ de la longitud total en ambos extremos;

Grupo C: Maderas que secan presentando deformaciones de $1-3 \%$ en cualquiera de sus dimensiones y rajaduras hasta $10 \%$ en cada uno de sus extremos.

\section{RESULTADOS}

EI Cuadro 3 presenta los resultados obtenidos en la velocidad de secado de las probetas de ensayo. El Cuadro 4 expone los resultados obtenidos en la evaluación de defectos y deformaciones en las probetas de madera.

\section{C uadro № 3. Velocidad de secado de cuatro maderas}

Madera

C ontenido de humedad

$\%$

Tiempo

Clasificación

Inicial Final

A tadijo

161

20

$30-45$

Rápido

Caucho masha

207

16

30-45

Rápido

Huamanzamana

158

16

30-45

Rápido

Topa

286

16

30-45

Rápido 


\section{C uatro № 4 Deformaciones de secado de cuatro maderas}

Madera

\section{Clasificación por tipo de deformación Arqueadura Encorvadura Torcedura Revirado}

$\begin{array}{lcccr}\text { A tadijo } & \text { A } & \text { A } & \text { A } & \text { A } \\ \text { Caucho masha } & \text { A } & \text { A } & \text { A } & \text { A } \\ \text { Huamanzamana } & \text { A } & \text { A } & \text { A } & \text { A } \\ \text { Topa } & \text { A } & \text { A } & \text { A } & \text { A }\end{array}$

\section{DISCUSION}

De acuerdo a los resultados obtenidos en el ensayo de secado al aire libre de cuatro maderas de rápido crecimiento procedentes de bosques secundarios, se encuentra que todas reducen rápidamente los contenidos de humedad por debajo del $20 \%$ en periodos no mayores a 120 días, lo que las califica como maderas de secado rápido.

A simismo, todas las maderas demuestran tener un buen comportamieto al secado natural en lo que se refiere a presencia de defectos, tales como grietas y rajaduras y a deformaciones, tal como arqueadura, encorvadura, revirado y torcedura.

El contenido de humedad inicial es, en todos los casos, muy alto, condición propia de madera procedente de árboles muy jóvenes y de especies de rápido crecimiento, caracterizadas por la presencia y abundancia de vasos de gran diámetro, indispensables para la activa circulación de sustancias nutritivas.

Las condiciones del ambiente de secado también han favorecido la rápida eliminación del agua libre y parte del agua higroscópica. En los meses de julio a octubre la humedad relativa promedio en Pucallpa es de $82 \%$ y la temperatura promedio es de $27^{\circ} \mathrm{C}$. En estas condiciones el aire tiene un déficit de saturación 
o capacidad para recibir vapor de agua eliminado de la madera en virtud a la energía calorífica transferida del medio para transformar el agua del estado líquido al de vapor.

La frecuencia e intensidad de deformaciones se ha minimizado debido, entre otros factores, a un adecuado apilado, con una correcta distribución del peso, utilizando separadores perfectamente escuadrados, de escuadría apropiada, convenientemente separados y conservando la alineación en toda la altura de la pila.

De igual forma, la práctica de estacionar la madera bajo techo, evitando la radiación solar directa, lo que acelera la velocidad de salida de la humedad, y el sellado de los extremos con pintura de esmal te sintético, que reduce la velocidad de secado a través del sistema vascular longitudinal, reducen la formación de grietas y rajaduras cuya causa es la violenta salida el agua libre, generando esfuerzos de tracción perpendicular a la fibra mucho mayores a la resistencia de la madera.

Los resultados obtenidos en el secado de la madera de caucho masha y huamanzamana coinciden con los obtenidos por A róstegui (1975) en ensayos efectuados al aire libre y sin cobertura en la Facultad de Ciencias Forestales de la UNALM, zona con humedad relativa promedio de $75 \%$ y temperatura que fluctúa entre $14-26^{\circ} \mathrm{C}$ dependiendo de la época del año. Según los estudios de la estructura anatómica efectuados por este autor y sus colaboradores, ambas maderas presentan poros de medianos a grandes, fibras de longitud media, pared celular delgada y lumen amplio, caracteres anatómicos que favorecen la rápida circulación de agua por acción de la gradiente de humedad.

EI análisis de la estructura anatómica de las maderas de huamazamana y topa efectuados por Valderrama et. al. (1986) y Detienne et. al. (1983), respectivamente, indican la presencia de poros difusos y de diámetro grande, elementos que favorecen la rápida evacuación del agua. 


\section{CONCLUSIONES}

De acuerdo a las condiciones del ensayo, dimensiones de probetas y condiciones ambientales, las maderas de Trema micrantha (atadijo), Sapium marmieri Hubert (caucho masha), J acaranda copaia A ube D. Don (huamanzamana) y Ochoroma pyramidale Swartz (topa) se comportan bien al secado natural, alcanzando contenidos de humedad inferiores al 20\% en menos de 120 días y sin formación de defectos ni deformaciones.

\section{BIBLIOGRAFIA}

AROSTEGUI V., A. et al. 1975. Estudio tecnológico de las maderas del Perú.Tomo II. Ensayos tecnológicos. Métodos y procedimientos. UNAM A. Lima. $152 \mathrm{p}$.

1975. Estudio tecnológico de las maderas del Perú. Tomo III. Características tecnológicas y usos de la madera de 40 especies del Bosque Nacional Alexander von Humbolt. UNA-M A. Lima. $174 \mathrm{p}$.

1979. Estudio integral de la madera para la construcción. DGFF/UNA. Lima. $149 \mathrm{p}$.

DETIENNE P. et al. 1983. Estudio anatomico do lenho da familia bombacaceae da America. In: Acta Amazonica XIII (5-6): pp. 831 - 868.

JUNTA DEL ACUERDO DE CARTAGENA. 1984. Manual de secado de las maderas del Grupo Andino. Lima. $549 \mathrm{p}$.

GONZALES F., R. 1970. Preservación y secado de la madera. Convenio UNA-MEM. Lima. $81 \mathrm{p}$.

HILDEBRANT, R. 1962. El secado de la madera aserrada. R. Hildebrant $\mathrm{M}$ aschimenban GM BH. Oberboinbengenwrt. $168 \mathrm{p}$.

HUNT, G. GARRAT, P. 1966. Preservación de maderas. Salvat Editores. M éxico. $596 \mathrm{p}$.

VALDERRAMA， H. et al. 1986. Estudio anatómico y clave de identificación de veinte especies forestales de la zona de Colonia Angamos, Rió Yavari y J enaro Herrera. UNAP-IIAP. Iquitos. 110 p. 